\title{
OTIMIZAÇÃO DE MÉTODOS DE REGRESSÃO MULTIVARIADA PARA QUANTIFICAÇÃO DE SULFAMETOXAZOL E TRIMETOPRIMA EM MEDICAMENTOS
}

\section{OPTIMIZATION OF MULTIVARIATE REGRESSION METHODS FOR QUANTIFICATION OF SULPHAMETHOXAZOLE AND TRIMETHOPRIM IN PHARMACEUTICAL FORMULATIONS}

\section{Fabiana Ernestina Barcellos da Silva}

Professora Titular

Universidade Regional Integrada do Alto Uruguai e das Missões Erechim

Departamento de Ciências da Saúde

Av. Sete de Setembro, 1621, Bairro Fátima, Erechim, RS, CEP: 99700-000

(54)35209000 fabiana@uri.com.br

\section{Gustavo Post Sabin}

Universidade de Santa Cruz do Sul

Mestrado em Sistemas e Processos Industriais

Av. Independência 2293 sala 5340, Santa Cruz do Sul, RS, CEP 96815-900

(51) 37177632 gustavosabin@yahoo.com.br

Edson Irineu Müller

Professor

Universidade Franciscana - UNIFRA

Departamento de Química

Rua dos Andradas, 1614, Bairro Centro, Santa Maria, RS, CEP: 97010-032

(55)32201200 edson_muller@yahoo.com.br 


\title{
Graciele Parisotto
}

\author{
Universidade Federal de Santa Maria
}

Departamento de Química

Av. Roraima, 1000, Cidade Universitária, Bairro Camobi, Santa Maria, RS, CEP: 97105900

(55) 3220-9445 gracieleparisotto@yahoo.com.br

\section{Érico Marlon de Moraes Flores}

Professor Associado

Universidade Federal de Santa Maria

Departamento de Química

Av. Roraima, 1000, Cidade Universitária, Bairro Camobi, Santa Maria, RS, CEP: 97105900

(55) 3220-9445 flores@quimica.ufsm.br

\section{Marco Flores Ferrão}

Professor Titular

Universidade de Santa Cruz do Sul

Departamento de Química e Física

Av. Independência 2293 sala 1211, Santa Cruz do Sul, RS, CEP 96815-900

(51)37177390ferrao@unisc.br

\section{RESUMO}

Neste trabalho foram desenvolvidos modelos de calibração por mínimos quadrados parciais (PLS) para a determinação simultânea de sulfametoxazol (SMZ) e trimetoprima (TMP) em medicamentos. Na busca de melhores modelos para a determinação destes princípios ativos em amostras comerciais foi empregado o método por mínimos quadrados parciais por intervalo ( $i$ PLS), identificando também qual(is) região(ões) do espectro no infravermelho apresentam melhor correlação com cada um dos princípios ativos estudados. Um total de 52 amostras sintéticas e 13 comerciais foi empregado para compor os conjuntos de calibração e de previsão, sendo consideradas as faixas de concentração de $400-900 \mathrm{mg} \mathrm{g}^{-1}$ para o sulfametoxazol e de $80-240 \mathrm{mg} \mathrm{g}^{-1}$ para a trimetoprima. Os dados espectrais foram adquiridos na faixa de $650-4000 \mathrm{~cm}^{-1}$ com resolução de $4 \mathrm{~cm}^{-1}$ por espectroscopia no 
infravermelho com transformada de Fourier empregando-se um acessório de reflexão total atenuada (FTIR/ATR). Tanto os modelos empregando toda a região espectral, como modelos construídos com apenas um intervalo do espectro apresentaram bons resultados para a previsão de ambos os princípios ativos. Os resultados empregando as técnicas de regressão PLS e $i$ PLS se mostraram promissores no desenvolvimento de metodologias mais simples, rápidas e não destrutivas para a determinação de sulfametoxazol e trimetoprima em formulações farmacêuticas, sendo estas viáveis para o controle de qualidade da indústria farmacêutica.

Palavras-chave: otimização, reflexão total atenuada, $i \mathrm{PLS}$, medicamentos, quimiometria.

\begin{abstract}
A partial least-squares calibration (PLS) procedure in combination with infrared spectroscopy has been developed for simultaneous determination of sulphamethoxazole (SMZ) and trimethoprim (TMP) in powder raw material mixtures used for manufacturing commercial pharmaceutical products. Multivariate calibration modeling procedures using interval partial least squares ( $i$ PLS) were applied to select a spectral range that provided the lowest prediction error in comparison to the full-spectrum model. The experimental matrix was constructed using 52 synthetic samples and 13 commercial samples. The considered concentration ranges were 400 to $900 \mathrm{mg} \mathrm{g}^{-1} \mathrm{SMZ}$ and 80 to $240 \mathrm{mg} \mathrm{g}^{-1} \mathrm{TMP}$. Spectral data were recorded between 650 and $4000 \mathrm{~cm}^{-1}$ with a $4 \mathrm{~cm}^{-1}$ resolution by Fourier transform infrared spectroscopy (FTIR) coupled with attenuated total reflection (ATR) accessory. The proposed procedure was compared with conventional procedure by high performance liquid chromatography (HPLC) using commercial samples containing SMZ and TMP. Results shown that PLS regression model combined to ATR-FTIR is a relatively simple, rapid and accurate procedure that could be applied to the simultaneous determination of SMZ and TMP in routine quality control of powder mixtures. A root mean square error of prediction (RMSEP) of $26.97 \mathrm{mg} \mathrm{g}^{-1}$ for SMZ and $12.23 \mathrm{mg} \mathrm{g}^{-1}$ for TMP was obtained after selection of better interval by $i$ PLS.
\end{abstract}

Key-words: optimization attenuated total reflection, $i \mathrm{PLS}$, pharmaceutical formulations, chemometrics.

\title{
1. INTRODUÇÃO
}

As sulfonamidas estão entre os mais antigos grupos de compostos antimicrobianos. Dentre estes encontramos o sulfametoxazol (SMZ), [4-amino-N-(5-methyl-3-isoxazolyl)benzenesulphonamide], que em combinação com a trimetoprima (TMP), [5-(3, 4, 5trimethoxybenzyl) pyrimidine-2, 4-diyldiamine], têm emprego no tratamento de diversas infecções, como por exemplo, bronquites, otites, uretrites, bem como algumas enterites. Esta associação também tem sido utilizada na prevenção e tratamento da pneumonia cujo agente é 
o Pneumocystis carinii (THE MERCK Index, 2001). O sulfametoxazol é uma sulfonamida com estrutura química semelhante ao ácido p-aminobenzóico (PABA) que inibe a incorporação deste no ácido fólico. Por outro lado, a trimetoprima evita a redução do dihidrofolato para tetrahidrofolato, o qual é essencial na síntese do DNA bacteriano. (HARDMAN et al., 1996; RANG et al., 2001).

Diversos métodos analíticos têm sido desenvolvidos para a determinação de sulfametoxazol (Figura 1a) e trimetoprima (Figura 1b) em formulações farmacêuticas. Nesta quantificação têm sido empregados métodos simples, através da espectrofotometria, baseados na formação de um derivado avermelhado pela diazotação de sulfonamidas (NAGARAJA et al., 2002), por sistemas de injeção em fluxo (FAN et al., 2003; FERNANDEZ DE CORDOVA et al., 2003; TOMSU et al., 2004), através de cromatografia líquida micelar de alta eficiência (GARCIA-ALVAREZ-COQUE et al., 1995), bem como outros métodos empregando cromatografia líquida (AKAY \& OZKAN, 2002), espectrofotometria derivativa (GRANERO et al., 2002), e ainda por voltametria (CARAPUCA et al., 2005).

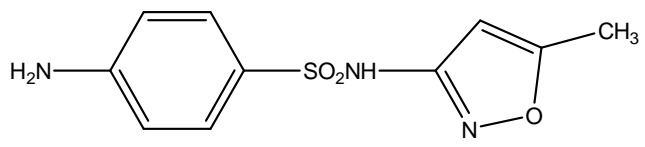

(a)

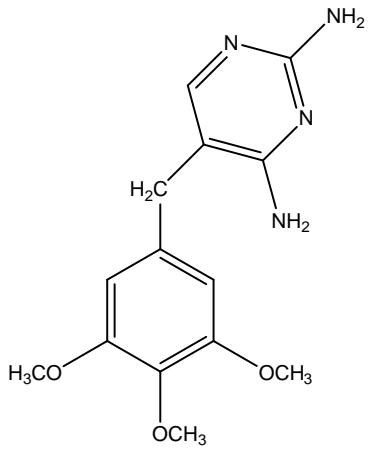

(b)

Figura 1: Fórmulas estruturais do sulfametoxazol (a) e trimetoprima (b).

Fonte: THE MERCK Index (2001)

Neste contexto, os métodos por reflectância difusa no infravermelho próximo (NIR) e médio (DRIFTS) vêm sendo apontados como alternativas às metodologias oficiais, em função destes métodos espectroscópicos não empregarem reagentes químicos, serem rápidos, não destrutivos e utilizarem poucos miligramas de amostra para a sua realização (DE SOUZA 
\& FERRÃO, 2006). A técnica NIR tem sido extensamente investigada nestes últimos anos na análise de medicamentos (PASQUINI, 2003; SUN et al., 2004; BRAGA \& POPPI, 2004; BODSON et al., 2006), entretanto a técnica DRIFTS tem sido pouco explorada (LUNDSTEDT-ENKEL et al., 2003; PARISOTO et al., 2005; PARISOTO et al., 2007), assim como a reflexão total atenuada (ATR). A espectroscopia no infravermelho com transformada de Fourier (FTIR) permite obter informações qualitativas e quantitativas rapidamente e, quando associada à reflexão total atenuada (ATR), simplifica a realização das análises (BORIN \& POPPI, 2005; GODOY et al., 2007). Na técnica por ATR, a amostra é colocada em contato com um elemento de reflexão interna (IRE) construído de um material com alto índice de refração como, por exemplo, seleneto de zinco ( $\mathrm{ZnSe}$ ). Na Figura 2 é apresentado um esquema de como o processo de reflexão interna ocorre (MIRABELLA, 1985).

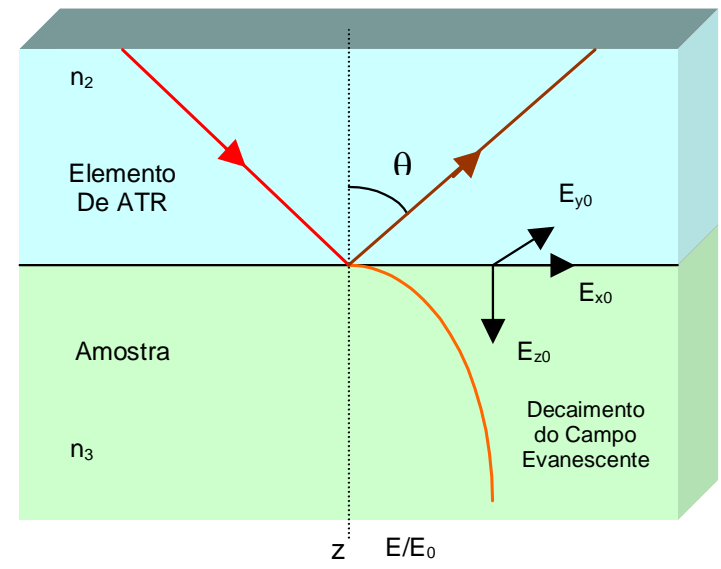

Figura 2: Representação da propagação da radiação infravermelha através do IRE.

Fonte: Ferrão (2000, p.46)

Por outro lado, a regressão por mínimos quadrados parciais (PLS) é a técnica mais comum de calibração multivariada para construção de modelos usando sinais de espectros (GELADI \& KOWALSKI, 1986). Em 1998, Spiegelman et al., demonstraram que a seleção da região espectral a ser modelada pode melhorar o desempenho dessa técnica de calibração em relação aos modelos que empregam o espectro inteiro. Regiões específicas (ou sinais infravermelhos) são selecionadas onde a colinearidade não está tão presente, gerando modelos 
mais robustos. Na prática, a otimização dos modelos de regressão multivariados, está baseada na identificação de subconjuntos dos dados completos (sub-regiões espectrais), que combinados ou não, minimizam os erros de predição.

O objetivo principal deste trabalho foi desenvolver modelos de regressão multivariada, utilizando dados de FTIR-ATR, para quantificar sulfametoxazol (SMZ) e trimetoprima (TMP) em formulações farmacêuticas. Para tanto, modelos de calibração utilizando toda a região espectral analisada (PLS) ou sub-regiões destas ( $i$ PLS) foram construídos e comparados, sendo apresentados os resultados mais representativos.

\section{REGRESSÃO POR MÍNIMOS QUADRADOS PARCIAS (PLS)}

No PLS a calibração multivariada é realizada utilizando a informação do espectro inteiro para construir um modelo da regressão, relacionado à propriedade de interesse. Por esta razão é chamado de método full-spectrum (BORIN e POPPI, 2005). Neste trabalho o PLS foi utilizado em ambiente MATLAB ${ }^{\circledR}$, o qual integra computação matemática, visualização e linguagem eficiente em ambiente flexível para computação técnica.

$\mathrm{Na}$ modelagem por mínimos quadrados parciais, tanto a matriz das variáveis independentes $\mathbf{X}$, como a das variáveis dependentes $\mathbf{Y}$ são representadas pelos escores e pelos pesos, conforme as expressões a seguir:

$$
\begin{aligned}
& \mathbf{X}=\mathbf{T} \mathbf{P}^{\mathbf{t}}+\mathbf{E} \\
& \mathbf{Y}=\mathbf{U} \mathbf{Q}^{\mathbf{t}}+\mathbf{F}
\end{aligned}
$$

A relação entre as duas matrizes de dados $\mathbf{X}$ e $\mathbf{Y}$ pode ser obtida, correlacionando-se os escores de cada bloco, a fim de obter uma relação linear descrita na seguinte expressão:

$$
\mathbf{U}=\mathbf{b} \mathbf{T}+\mathbf{e}
$$


Onde: U é uma matriz contendo as propriedades (variáveis dependentes) de todas estas amostras; b é um vetor contendo os parâmetros do modelo; T é uma matriz de resposta ( como um conjunto de espectros) para uma série de amostras de calibração e e é um vetor que representa o ruído do espectro e os erros do modelo (KONZEN et al., 2003).

\subsection{Regressão por mínimos quadrados parciais por intervalo (iPLS)}

Existem várias maneiras de selecionar a região espectral utilizando critérios objetivos, como por exemplo, avaliar a correlação da região selecionada com a o método de referência $\left(\mathrm{R}^{2}\right)$, ou avaliar a raiz quadrada dos erros médios de previsão (RMSEP), e ainda utilização de algoritmos que indicam o conjunto de variáveis espectrais que poderá dar melhores resultados com base no $\mathrm{R}^{2}$ e/ou no RMSEP. Dentre os algoritmos mais empregados encontram-se os métodos heurísticos como o algoritmo genético e a busca tabu, ou determinísticos como, por exemplo, o método de mínimos quadrados parciais por intervalo (Interval Partial Least-Squares - $i$ PLS).

O método iPLS é uma extensão do PLS (NORGAARD et al., 2000), no qual dividese o conjunto de dados (espectros) em um determinado número de intervalos, constrói-se o modelo PLS para cada intervalo, apresentando os resultados em um gráfico para facilitar a comparação. O método é planejado para dar uma visão geral dos dados e pode ser útil na interpretação de quais sinais do espectro são mais representativos na construção de um bom modelo de calibração.

\section{MATERIAIS E MÉTODOS}

\subsection{Amostragem}

Para a obtenção de amostras sintéticas foram utilizados sulfametoxazol e trimetoprima adquiridos da empresa Henrifarma (São Paulo, Brasil) e amido e estearato de magnésio, com pureza farmacêutica, como excipientes. Para a realização das análises 
cromatográficas foram empregados metanol, acetonitrila e trietilamina grau HPLC. Além das amostras sintéticas foram utilizadas 13 amostras comerciais, de diferentes laboratórios, adquiridas no comércio local. Todas as amostras foram misturadas e/ou trituradas em moinho criogênico Spex Certiprep (model 6750 Freezer Mill, Metuchen, EUA).

\subsection{Método de referência}

A análise do sulfametoxazol e da trimetoprima foi realizada por cromatografia líquida de alta eficiência (HPLC) seguindo o método de referência USP 28 (UNITED STATES PHARMACOPOEIA, 2004). Para a aquisição dos cromatogramas e quantificação dos princípios ativos, foi utilizado um sistema de cromatografia líquida da Agilent 1100 Series equipado com uma bomba Agilent modelo G1311A, detector Agilent modelo G1315B DAD e amostrador automático Agilent modelo G133A ALS. Foi selecionado o comprimento de onda de $254 \mathrm{~nm}$ para as leituras, e as áreas dos picos foram integradas, usando o programa CHEMSTATION que acompanha o equipamento. As substâncias químicas de referência (TMP e SMZ) foram gentilmente cedidas pela Farmacopéia Brasileira.

\subsection{Obtenção dos espectros no infravermelho e modelagem dos dados}

Todos os espectros foram obtidos na faixa de $4000-650 \mathrm{~cm}^{-1}$, com resolução de 4

$\mathrm{cm}^{-1}$ e 16 varreduras utilizando um espectrômetro Perkin Elmer modelo Spectrum One FTIR, sendo acoplado um acessório ATR com cristal de ZnSe. Os espectros foram obtidos em

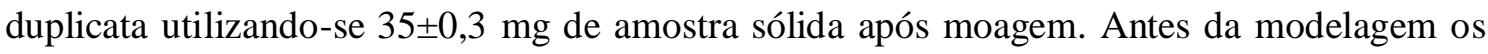
dados foram tratados com correção de espalhamento de luz (MSC) e como pré-processamento os dados foram auto-escalados.

Os dados espectrais (matriz $\mathrm{X}$ ) e os valores obtidos para cada princípio ativo por HPLC (vetores Y) foram modelados usando ambiente MATLAB versão 6.1 (The Math Works, Natick, USA). A rotina PLS foi realizada através do PLS Toolbox versão 3.5 (Eigenvector Technologies, Manson, USA). O iToolbox para MATLAB (NORGAARD et al., 
2000) foi usado para desenvolver os modelos $i$ PLS, sendo divididos os espectros em 10, 25 e 50 intervalos para realizar as comparações. Os programas foram rodados num microcomputador IBM - compatível Intel Pentium 4 CPU $3.00 \mathrm{GHz}$ com 1 Gb de RAM.

\subsection{Avaliação dos modelos de calibração multivariada}

Para calcular o erro dos modelos de calibração foi empregado o RMSE (Root Mean Square Error), conforme equação 1, onde n é o número de espectros, $\mathrm{y}_{\mathrm{i}}$ e $\hat{\mathrm{y}}_{\mathrm{i}}$ são os valores determinados pelo método oficial e predito pelo modelo do PLS (ou $i$ PLS), respectivamente, utilizando as amostras do conjunto de calibração para validação cruzada (RMSECV) ou de previsão externa (RMSEP).

$$
R M S E=\sqrt{\frac{\sum_{i=1}^{n}\left(y_{i}-\hat{y}_{i}\right)^{2}}{n}}
$$

\section{RESULTADOS E DISCUSSÃO}

\subsection{Modelos PLS e iPLS para a SMZ}

Os resultados dos melhores modelos para a previsão da SMZ, empregando toda a região espectral (modelo PLS) e partes do espectro (modelos $i$ PLS), são apresentados na Tabela 1. Os valores de RMSECV, $\mathrm{R}_{\text {cal }}^{2}$ e RMSEP são semelhantes para os modelos representados, exceto para o caso que empregou um único intervalo (de número 43), quando o espectro foi divido em 50 intervalos. Dentre estes modelos podemos selecionar aquele que foi construído com o intervalo número 23, empregando-se 6 variáveis latentes, quando da divisão do espectro em 25 partes. A figura 3 ilustra o comportamento dos modelos obtidos para cada um dos 25 intervalos, juntamente com o modelo global. Nesta figura a linha tracejada representa o erro de validação cruzada para o espectro total, assim como cada barra o erro associado ao emprego de um único intervalo. Os números em itálico referem-se ao número de variáveis latentes utilizado na construção de cada modelo $i \mathrm{PLS}$, sendo neste caso empregadas 4 variáveis latentes para o modelo global. Uma análise da Figura 3 nos permite verificar que 
os melhores modelos $i \mathrm{PLS}$ foram àqueles cujo erro de validação cruzada é inferior a $60 \mathrm{mg}$ de $\mathrm{SMZ} \mathrm{g}^{-1}$, correspondendo aos intervalos 4, 5, 7,19-25, sendo que o melhor intervalo foi o 23 (Figura 4), que apresentou RMSECV próximo ao modelo global.

Tabela 1: Resultados dos modelos de regressão PLS e $i$ PLS para a previsão da SMZ.

\begin{tabular}{|c|c|c|c|c|c|c|}
\hline Modelo & $\mathbf{N} \mathbf{V}^{\mathrm{a}}$ & Intervalos & $V L s^{b}$ & $\begin{array}{c}\text { RMSECV }^{\mathrm{c}} \\
\left(\mathrm{mg} \mathrm{de} \mathrm{SMZ.g}^{-1}\right)\end{array}$ & $\mathbf{R}_{\text {cal }}^{2}$ & $\begin{array}{c}\text { RMSEP }^{\mathrm{d}} \\
\left(\mathrm{mg} \mathrm{de} \mathrm{SMZ.g}^{-1}\right)\end{array}$ \\
\hline PLS & 3351 & todos & 4 & 38,18 & 0,9404 & 26,78 \\
\hline$i$ PLS10 & 335 & 2 & 7 & 39,19 & 0,9376 & 26,46 \\
\hline$i$ PLS25 & 135 & 23 & 6 & 37,80 & 0,9421 & 26,97 \\
\hline$i$ PLS50 & 68 & 43 & 4 & 71,43 & 0,7994 & 26,93 \\
\hline
\end{tabular}

${ }^{\mathrm{a}} \mathrm{NV}$ : número total de variáveis

${ }^{\mathrm{b}}$ VLs: número de variáveis latentes

${ }^{\mathrm{c}}$ RMSECV: Root Mean Square Error of Cross Validation

${ }^{\mathrm{d}}$ RMSEP: Root Mean Square Error of Prediction (usando o conjunto de previsão)

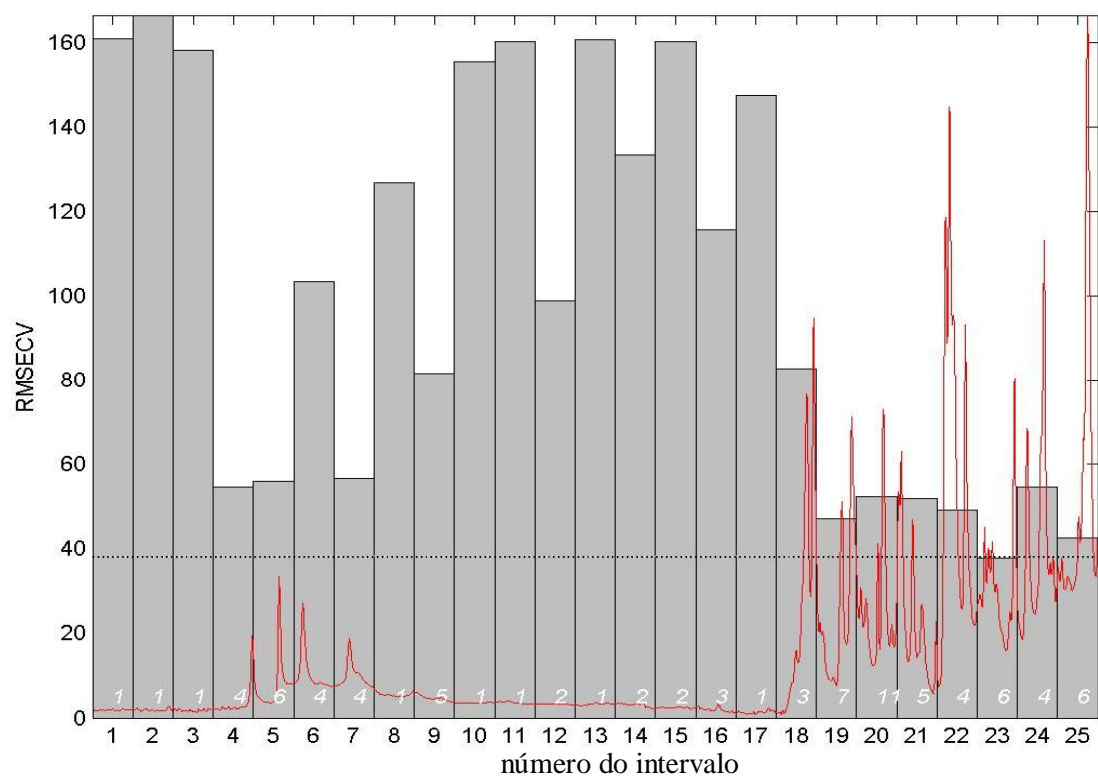

Figura 3: Erros de validação cruzada para o modelo PLS (linha tracejada) e para os 25 modelos $i$ PLS (barras) para a determinação do sulfametoxazol. 


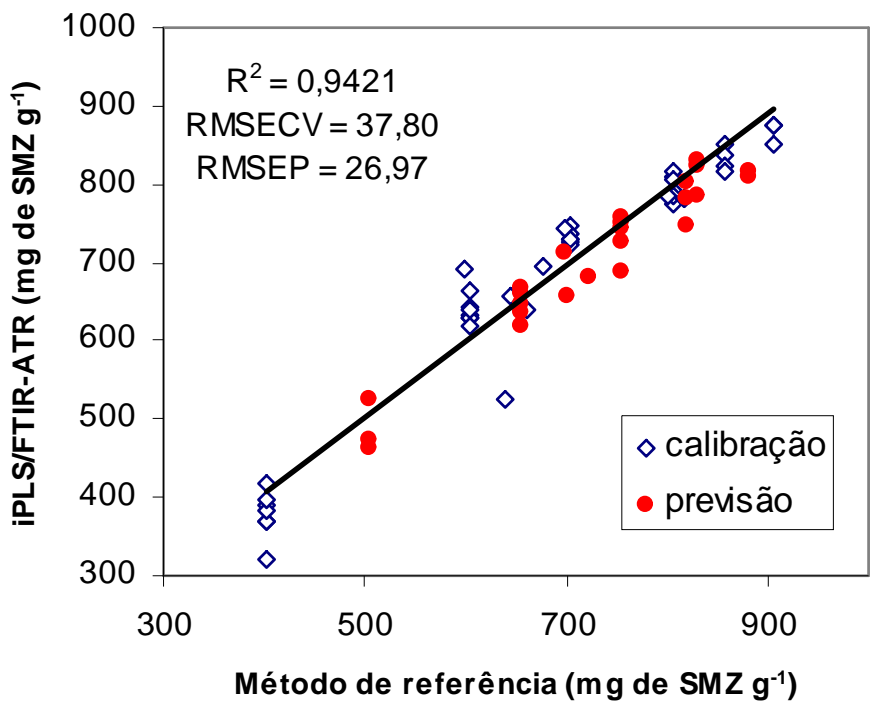

Figura 4: Gráfico dos valores de referência versus valores previstos para o sulfametoxazol pelo modelo utilizando o intervalo 23, com 6 variáveis latentes, sendo o espectro dividido em 25 intervalos.

\subsection{Modelos PLS e $i$ PLS para a TMP}

Da mesma forma, os resultados dos melhores modelos para a previsão da TMP, empregando toda a região espectral (modelo PLS) e partes do espectro (modelos $i$ PLS), são apresentados na Tabela 2. Neste caso todos valores de RMSECV, $\mathrm{R}_{\text {cal }}^{2}$ e RMSEP são semelhantes. Dentre estes modelos podemos selecionar aquele que foi construído com o intervalo número 25, empregando-se 9 variáveis latentes, quando da divisão do espectro em 25 partes, conforme Figura 5. 
Tabela 2: Resultados dos modelos de regressão PLS e $i$ PLS para a previsão da TMP.

\begin{tabular}{|c|c|c|c|c|c|c|}
\hline Modelo & $\mathbf{N V}^{\mathbf{a}}$ & Intervalos & VLs $s^{b}$ & $\begin{array}{c}\text { RMSECV } \\
\left(\mathrm{mg} \mathrm{de} \mathrm{TMP.g}^{-1}\right)\end{array}$ & $\mathbf{R}_{\text {cal }}^{2}$ & $\begin{array}{c}\text { RMSEP }^{\mathrm{d}} \\
\left(\mathrm{mg} \mathrm{de} \mathrm{TMP.g}^{-1}\right)\end{array}$ \\
\hline PLS & 3351 & todos & 4 & 16,51 & 0,8597 & 12,31 \\
\hline$i$ PLS10 & 335 & 2 & 5 & 16,60 & 0,8570 & 12,25 \\
\hline$i$ PLS25 & 135 & 25 & 9 & 15,61 & 0,8818 & 12,23 \\
\hline$i$ PLS50 & 68 & 42 & 5 & 15,57 & 0,8742 & 12,28 \\
\hline
\end{tabular}

${ }^{a} \mathrm{NV}$ : número total de variáveis

${ }^{\mathrm{b}}$ VLs: número de variáveis latentes

${ }^{c}$ RMSECV: Root Mean Square Error of Cross Validation

${ }^{\mathrm{d}}$ RMSEP: Root Mean Square Error of Prediction (usando o conjunto de previsão)

Através da curva de correlação, entre os valores do método de referência (HPLC) e valores previstos pelo método empregando os dados de FTIR-ATR, podemos verificar que tanto as amostras do conjunto de calibração, quanto às de previsão, apresentam bons resultados, indicando que o modelo tem boa capacidade preditiva para as amostras externas, não sendo verificado sobreajuste (overffiting).

Outro aspecto relevante que deve ser ressaltado trata-se dos resultados alcançados em ambos os casos (SMZ e TMP), indicando que não existe um ganho real na seleção de menores regiões espectrais para a modelagem. Isto é consistente, se as informações químicas necessárias às modelagens estão presentes nas variáveis latentes selecionadas na construção de cada modelo em questão. 
$\underline{\text { www.producaoonline.ufsc.br }}$

ISSN 1676 - 1901 / Edição especial/dezembro de 2007 Artigo selecionado dos anais - XXVII Encontro Nacional de Engenharia de Produção

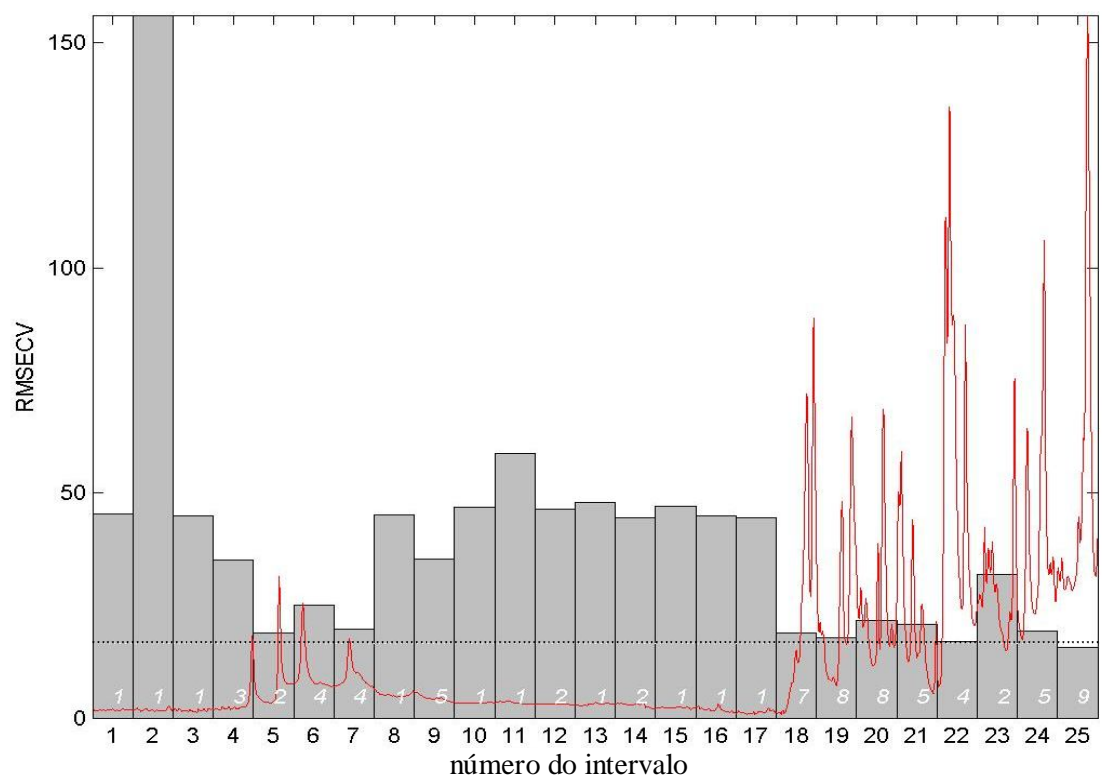

Figura 5: Erros de validação cruzada para o modelo PLS (linha tracejada) e para os 25 modelos iPLS (barras) para a determinação da trimetoprima.

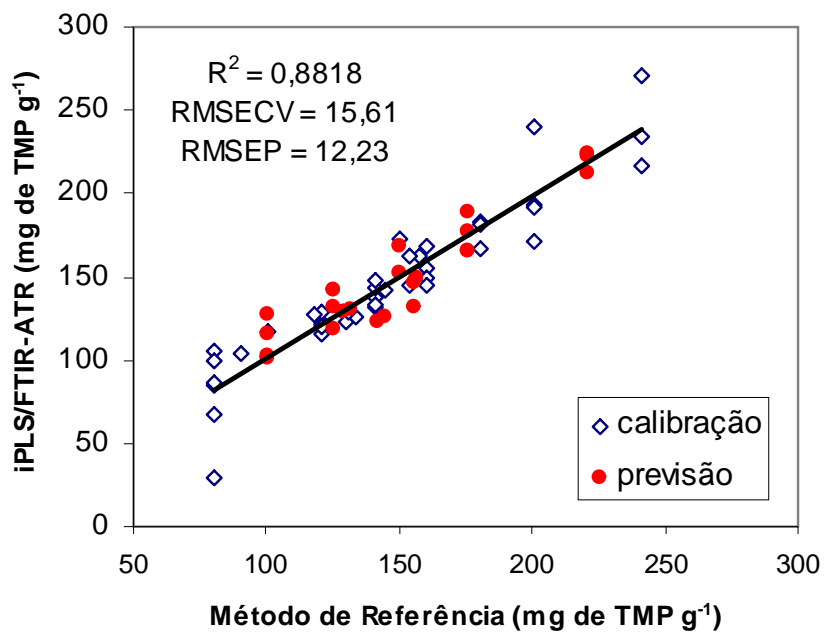

Figura 6: Gráfico dos valores de referência versus valores previstos para a trimetoprima pelo modelo utilizando o intervalo 25 , com 9 variáveis latentes, sendo o espectro dividido em 25 intervalos. 
Por outro lado, os melhores modelos $i$ PLS10 e $i$ PLS25 obtidos apresentam maior robustez frente aos modelos que empregam o espectro inteiro, em virtude de serem menos vulneráveis a problemas experimentais que por ventura ocorram na aquisição dos espectros, principalmente se considerarmos variações na umidade do ar ou da concentração do $\mathrm{CO}_{2}$ que resultariam em espectros com maior ruído, sendo este ruído menos representativo nas regiões selecionadas para os modelos $i \mathrm{PLS} 10$ e $i \mathrm{PLS} 25$ anteriormente tabelados.

\section{CONCLUSÕES}

O presente trabalho mostrou ser possível a construção de modelos de calibração multivariados, empregando dados de espectroscopia no infravermelho com transformada de Fourier com acessório de reflexão total atenuada (FTIR-ATR), para a determinação simultânea de sulfametoxazol (SMZ) e trimetoprima (TMP) em medicamentos comercializados no Brasil. Os resultados obtidos mostram que bons modelos podem ser obtidos tanto com os espectros por inteiro (PLS), como por determinadas regiões espectrais (iPLS) com $1 / 10$ ou $1 / 25$ do total das variáveis.

Constatou-se ainda, que a utilização do algoritmo $i$ PLS apresenta-se como uma boa alternativa para a otimização de modelos de calibração multivariados empregando espectros FTIR, gerando modelos com previsão equivalente ao PLS, porém com vantagem de utilizar somente sub-regiões espectrais mais correlacionadas, sendo menos vulneráveis que o PLS full-spectrum.

Por fim, os resultados empregando as técnicas de regressão PLS e iPLS aos dados FTIR-ATR se mostraram promissores no desenvolvimento de metodologias mais simples, rápidas e não destrutivas para a determinação de sulfametoxazol e trimetoprima em formulações farmacêuticas, sendo estas viáveis para o controle de qualidade da indústria farmacêutica. 


\section{AGRADECIMENTOS}

Os autores agradecem o apoio financeiro da CAPES e pela colaboração da Farmacopéia Brasileira.

\section{REFERÊNCIAS}

AKAY, C. \& OZKAN, S.A. Simultaneous LC determination of trimethoprim and sulphamethoxazole in pharmaceutical formulations. J. Pharm. Biomed. Anal. Vol. 30, n.4, p.1207-1213, 2002.

BODSON, C.; DEWE, W.; HUBERT, P. \& DELATTRE, L. Comparison of FT-NIR transmission and UV-vis spectrophotometry to follow the mixing kinetics and to assay low-dose tablets containing riboflavin. J. Pharm. Biomed. Anal. Vol. 41, n.3, p.783-790, 2006.

BORIN, A. \& POPPI, R.J. Application of mid infrared spectroscopy and iPLS for the quantification of contaminants in lubricating oil. Vib. Spect. Vol.37, p.27-32, 2005.

BRAGA, J.W. \& POPPI, R.J. Validação de modelos de calibração multivariada: Uma aplicação na determinação de pureza polimórfica de carbamazepina por espectroscopia no infravermelho próximo. Quím. Nova Vol. 27, p.1004-1011, 2004.

CARAPUCA, H.M.; CABRAL, D.J. \& ROCHA, L.S. Adsorptive stripping voltammetry of trimethoprim: mechanistic studies and application to the fast determination in pharmaceutical suspensions. J. Pharm. Biomed. Anal. Vol.38, n.2, p.364-369, 2005.

DE SOUZA, J.S. \& FERRÃO, M.F. Aplicações da espectroscopia no infravermelho no controle de qualidade de medicamentos contendo diclofenaco de potássio. Parte I: dosagem por regressão multivariada. Rev. Bras. Cienc. Farm. Vol.42, n.3, p.437-445, 2006.

FAN, J.; CHEN, Y.; FENG, S.; YE, C. \& WANG, J. Flow-injection spectrophotometric determination of sulfadiazine and sulfamethoxazole in pharmaceuticals and urine. Anal Sci. Vol.19, n.3, p.419-422, 2003.

FERNANDEZ DE CORDOVA, M.L.; ORTEGA BARRALES, P.; RODRIGUEZ TORNE, G. \& MOLINA DIAZ, A. A flow injection sensor for simultaneous determination of sulfamethoxazole and trimethoprim by using Sephadex SP C-25 for continuous on-line separation and solid phase UV transduction. J. Pharm. Biomed. Anal. Vol. 31, n.4, p.669677, 2003. 
FERRÃO, M.F. Aplicação de técnicas espectroscópicas de reflexão no infravermelho no controle de qualidade de farinha de trigo. Ph.D Thesis, Instituto de Química, Universidade Estadual de Campinas, Campinas, 2000.

GARCIA-ALVAREZ-COQUE，M.C.; SIMO-ALFONSO， E.F.; RAMIS-RAMOS， G. \& ESTEVE-ROMERO, J.S. High-performance micellar liquid chromatography determination of sulphonamides in pharmaceuticals after azodye precolumn derivatization. J. Pharm. Biomed. Anal. Vol. 13, n.3, p.237-245, 1995.

GELADI, P. \& KOWALSKI, B.R. Partial Least-Squares Regression: A Tutorial. Anal. Chim. Acta. Vol.185, p.1-17, 1986.

GODOY, S. C. ; FERRÃO, M. F. ; GERBASE, A. E. . Determination of Hydroxyl Value of Soybean Polyol by Attenuated Total Reflectance/Fourier Transform Infrared Spectroscopy. J. Am. Oil Chem. Soc., Vol. 84, p. 503-508, 2007.

GRANERO, G.; GARNERO, C. \& LONGHI, M. Second derivative spectrophotometric determination of trimethoprime and sulfamethoxazole in the presence of hydroxypropyl-beta-cyclodextrin (HP-beta-CD). J. Pharm. Biomed. Anal. Vol. 29, n.1-2, p.51-59, 2002.

HARDMAN, J.G.; LIMBIRD, L.E. \& GILMAN, A.G. (Ed.). As bases farmacológicas da terapêutica. $9^{a}$ ed., México: MacGraw-Hill Interamericana, 1996.

KONZEN, P.H.A.; FURTADO, J.C.; CARVALHO, C.W.; FERRÃO, M.F.; MOLZ, R.F.; BASSANI, I.A. \& HÜNING, S.L. Otimização de métodos de controle de qualidade de fármacos usando algoritmos genéticos e busca tabu. Pesquisa Operacional, v. 23, n. 1, Rio de Janeiro, jan/abr, 2003.

LUNDSTEDT-ENKEL， K.; GABRIELSSON， J.; OLSMAN， H.; SEIFERT, H.; PETTERSEN, J., LEK, P.M., BOMAN, A. \& LUNDSTEDT, T. Different multivariate approaches to material discovery, process development, PAT and environmental process monitoring. Chemom. Intell. Lab. Syst. Vol. 84, n.1-2, p.201-207, 2006.

MIRABELLA, F.M. Jr. Internal reflection spectroscopy. Appl. Spectrosc. Rev. Vol.21, p.45-178, 1985.

NAGARAJA, P.; SUNITHA, K.R.; VASANTHA, R.A. \& YATHIRAJAN, H.S. Iminodibenzyl as a novel coupling agent for the spectrophotometric determination of sulfonamide derivatives. Eur. J. Pharm. Biopharm. Vol. 53, n.2, p.187-192, 2002.

NORGAARD, L.; SAUDLAND, A.; WAGNER, J.; NIELSEN J. P.; MUNCK, L. \& ENGELSEN, S. B. Interval Partial Least-Squares Regression (iPLS): A Comparative Chemometric Study with an Example from Near-Infrared Spectroscopy. Appl. Spectrosc. Vol.54, n.3, p.413-419, 2000. 
PARISOTTO, G.; DE SOUZA, J.S.; FERRÃO, M.F.; FURTADO, J.C. \& MOLZ, R.F. Análise exploratória aplicada no estudo de medicamentos contendo piroxicam. Rev. Bras. Ciênc. Farm. Vol. 41, n.4, p.499-505, 2005.

PARISOTTO, G. ; FERRÃO, M. F. ; FURTADO, J. C. ; MOLZ, R. F. Determination of amoxicillin content in powdered pharmaceutical formulations using DRIFTS and PLS. Rev. Bras. Ciênc. Farm., Vol. 43, p. 89-96, 2007.

PASQUINI, C. Near infrared spectroscopy: fundamentals practical aspects and analytical applications. J. Braz. Chem. Soc. Vol.14, n.2, p.138-219, 2003.

RANG, H. P.; RITTER, J. M. \& DALE, M. M. Farmacologia. $4^{\text {a }}$ ed. Rio de Janeiro: Guanabara Koogan, 2001.

SPIEGELMAN, C. H.; MCSHANE, M. J.; COTÉ, G. L.; GOETZ, M. J.; MOTAMEDI, M. \& YUE Q. L. Theoretical justification of wavelength selection in PLS calibration: development of a new algorithm. Anal. Chem. Vol.70, p.35-44, 1998.

SUN, M.L.; XIANG, B.R. \& AN, D.K. A near-infrared diffuse reflectance analysis method for the noninvasive quantitative analysis of ambroxol hydrochloride tablets. Yao Xue Xue Bao Vol. 39, n.1, p.60-63, 2004.

THE MERCK Index: An encyclopedia of chemicals, drugs, and biologicals. $13^{\mathrm{a}} \mathrm{ed}$. New Jersey: MERCK, 2001.

TOMSU, D.; CATALA ICARDO, M. \& MARTINEZ CALATAYUD, J. Automated simultaneous triple dissolution profiles of two drugs, sulphamethoxazole-trimethoprim and hydrochlorothiazide-captopril in solid oral dosage forms by a multicommutation flow-assembly and derivative spectrophotometry. J. Pharm. Biomed. Anal. Vol. 36, n.3, p.549-557, 2004.

UNITED STATES PHARMACOPOEIA 28a․ Rockville, United States Pharmacopeial Convention, 2004. 